\title{
Influence of narrowband ultraviolet-B phototherapy on plasma concentration of matrix metalloproteinase-12 in psoriatic patients
}

\author{
Edyta K. Głażewska', Marek Niczyporuk ${ }^{1}$, Andrzej Przylipiak' ${ }^{1}$, Maciej Szmitkowski², Monika Zajkowska², \\ Ewa Będkowska ${ }^{3}$, Robert Terlikowski ${ }^{4}$, Stawomir Ławicki
}

${ }^{1}$ Department of Esthetic Medicine, Medical University of Bialystok, Bialystok, Poland

${ }^{2}$ Department of Biochemical Diagnostics, Medical University of Bialystok, Bialystok, Poland

${ }^{3}$ Department of Haematological Diagnostics, Medical University of Bialystok, Bialystok, Poland

${ }^{4}$ Department of Rehabilitation, Medical University of Bialystok, Bialystok, Poland

Adv Dermatol Allergol 2017; XXXIV (4): 328-333

DOI: https://doi.org/10.5114/ada.2017.69312

\begin{abstract}
Introduction: Matrix metalloproteinase-12 (MMP-12) may play an important role in the pathogenesis and spread of psoriatic disease.

Aim: To investigate plasma levels of the selected enzyme in plaque psoriasis patients before and after the course of narrowband UVB (NBUVB) therapy with respect to disease advancement.

Material and methods: The cohort included 49 patients suffering from plaque psoriasis, divided into groups according to severity of the disease. The control group consisted of 40 healthy volunteers. Plasma levels of MMP-12 were determined using immunoenzyme assay (ELISA), while the Psoriasis Area and Severity Index (PASI) was used to define disease advancement.

Results: The results have shown a significantly decreased plasma level of MMP-12 in the total psoriasis patient group compared to healthy individuals, declining with the increase in disease advancement. The NBUVB therapy caused a decrease in the concentration of the analyzed enzyme, but this change was not statistically significant in the total group of psoriatic patients, while a significant change was detected in patients with a mild advancement of the disease.

Conclusions: Decreased synthesis of MMP-12 may lead to the stimulation of the epidermal angiogenesis process, which results in the appearance and spread of psoriatic scales. Based on the obtained results, macrophage metalloelastase seems to be a negatively reacting plasma biomarker of the studied disease.
\end{abstract}

Key words: matrix metalloproteinase-12, psoriasis, narrowband ultraviolet-B phototherapy.

\section{Introduction}

Psoriasis is the most common chronic inflammatory skin disease driven by the activated immune system. This skin pathology affects approximately $3 \%$ of the global population, depends on geographic location and has relatively same frequency in both genders [1]. First symptoms of psoriasis may appear at every age, but there are two peaks in the age-specific incidence rate: the first at the age of 20-30 (type I) and the second near the age of 60 (type II) [2]. The analyzed condition affects not only patients' appearance, but has also a harmful impact on their frame of mind. If the disease manifests once, the individual suffers from it during the whole life with episodes of psoriatic lesion eruptions and remission for no clear reason [3].

Abnormal keratinocyte differentiation and hyperproliferation, leukocyte infiltrations to dermis and blood vessel hyperplasia are the most characteristic histopathological symptoms of psoriasis [4]. The exact etiology of this disease is still not fully understood. It has been assumed that the cause of its occurrence can be associated with genetic predisposition and environmental triggering factors and it may also be related to other disorders [5].

Address for correspondence: Edyta K. Głażewska, Department of Esthetic Medicine, Medical University of Bialystok, 3 Akademicka St, 15-267 Bialystok, Poland, fax: +48 698909 477, e-mail: kasiaglazewska@wp.pl Received: 10.05.2016, accepted: 1.06.2016. 
The already performed analyses suggest many indicators involved in psoriasis development. One of them are metalloproteinases (MMPs) - a family of zinc-dependent enzymes responsible for the stability of extracellular matrix (ECM). The proper level of those parameters is essential to maintain tissue homeostasis and skin cell function in extreme conditions. The remarkable role of the above-mentioned factors in psoriasis pathogenesis includes for instance the ECM and basal membrane (BC) macromolecules cleaving, stimulation of cell migration, tissue remodeling or epithelial apoptosis. Upregulation of only MMP-12, also known as macrophage metalloelastase, may lead to ECM and BC modification and also the activation of other matrix metalloproteinases, which can result in disease progression [6-9].

Broad-band (BB) UV-B alone (wavelengths between 280 and $320 \mathrm{~nm}$ ) for the treatment of psoriasis was used for the first time in the 1970s, while narrowband ultraviolet-B (NBUVB) was introduced in 1988. Phototherapy is the second-line treatment of psoriasis, recommended when the typical therapy fails or is contraindicated or impractical. Phototherapy may result in the clearance of disease symptoms after just 5-8 weeks. This kind of treatment had the highest satisfaction rates in psoriasis lesion improvement [10].

\section{Aim}

The aim of this research was to determine the plasma levels of MMP-12 in psoriatic patients and the influence of NBUVB phototherapy course on the concentration of that enzyme. We also conducted this study to examine the association between tested human macrophage metalloelastase levels and psoriasis severity.

The data obtained in this paper may bring the information about suspected correlations of MMP-12 and psoriasis pathogenesis, advancement and its influence on the lesion improvement during a successful treatment.

\section{Material and methods}

Table 1 shows the tested groups. The study included 49 plaque psoriasis patients whose condition was diagnosed and treated in the Department of Dermatology, Division of Outpatient Care, University Hospital, Bialystok, Poland, between 2013 and 2015. Assigning the participants to specific groups of disease advancement was carried out based on the Psoriasis Area and Severity Index (PASI) formulated by Fredrikson and Pettersson [11]. All patients underwent a course of narrow-band ultraviolet-B phototherapy composed of 20 sessions. Considering the fact that this kind of treatment is suitable for patients with mild and medium psoriasis advancement, the study included the patients with PASI score of 2-30.
The control group consisted of 40 healthy volunteers who had never suffered from any autoimmunological or chronic dermatological diseases.

Moreover, a key exclusion criterion in both groups was any additional pharmacotherapy.

All patients gave their informed consent to the participation in the study. The research was approved by the Biochemical Committee of the Medical University of Bialystok, approval number R-I-002/74/2014

\section{Radiation source}

The whole body irradiation was performed in a Cosmedico GP-36 phototherapy unit (Cosmedico Medical System, Stuttgart, Germany), equipped with NBUVB TL-01 100W tubes (HEINE.MED, Baden-Württemberg, Germany). The energy output was measured with a standard intrinsic UV-meter.

\section{Phototherapy protocol}

Individual minimum erythema doses (MEDs) of NBUVB were determined before starting the exposures to prevent burns. The patients underwent $2-3$ treatments weekly (to the total of 20), starting with a NBUVB dose of $0.018-0.025 \mathrm{~J} / \mathrm{cm}^{2}$, and increasing by approximately 10-20\% per week, up to a maximum dose of $2.015 \mathrm{~J} / \mathrm{cm}^{2}$.

\section{Biochemical analysis}

Venous blood samples were collected from each patient twice - before the beginning and after the last $-20^{\text {th }}$ - NBUVB phototherapy treatment into a heparin sodium tube, centrifuged for $15 \mathrm{~min}$ at $1000 \times \mathrm{g}$ to obtain plasma samples and stored at $-85^{\circ} \mathrm{C}$ until assayed. The tested cytokine (MMP-12) were measured using EIAab (Quantikine

Table 1. Characteristics of psoriatic patients and the control group

\begin{tabular}{|c|c|c|c|}
\hline \multicolumn{3}{|l|}{ Study group } & Number of patients \\
\hline \multicolumn{3}{|c|}{ Psoriasis patients: } & $\begin{array}{c}49 \text { (23 females, } \\
26 \text { males) }\end{array}$ \\
\hline \multicolumn{2}{|l|}{ Type } & Plaque type & 49 \\
\hline \multicolumn{3}{|c|}{ Median age (range) [years] } & $39(18-76)$ \\
\hline \multirow[t]{5}{*}{ Severity: } & \multirow[t]{3}{*}{ Mild } & & 34 \\
\hline & & $\mathrm{PASI}<5$ & 15 \\
\hline & & PASI 5-10 & 19 \\
\hline & \multirow[t]{2}{*}{ Medium } & & 15 \\
\hline & & $\begin{array}{c}\text { PASI } \\
>10-<30\end{array}$ & 15 \\
\hline \multicolumn{3}{|c|}{ Healthy subjects: } & $\begin{array}{l}40 \text { (19 females, } \\
21 \text { males) }\end{array}$ \\
\hline \multicolumn{3}{|c|}{$\begin{array}{l}\text { Median age (range) } \\
\text { [years] }\end{array}$} & $36(18-75)$ \\
\hline
\end{tabular}


Human MMP-12 Immunoassay, Wuhan EIAab Science Co., Wuhan, China), according to the manufacturer's protocols. Duplicate samples were assessed for each patient.

The intra-assay coefficient of variation (CV\%) of MMP-12 is reported to be $\leq 4.7 \%$, while the inter-assay coefficient of variation (CV\%) $-\leq 7.3 \%$.

The assay showed no significant cross-reactivity or interference with numerous human cytokines and other growth factors.

\section{Statistical analysis}

The statistical analysis was performed using Program Statistica 12.0 PL. A preliminary statistical analysis revealed that the MMP-12 failed to follow a normal distribution. Consequently, the Mann-Whitney U-test was used for statistical analysis between psoriatic patients and the control group. Additionally, statistical analysis between the groups with different advancement of psoriasis was performed with the use of Bonferroni correction. Moreover, the Wilcoxon matched pair test was used for statistical analysis of changes in parameters between the beginning and the end of the NBUVB phototherapy treatment. The data were presented as a median, mean and range. The Spearman rank correlation was used in the correlation analysis. Statistically significant differences were defined as comparisons resulting in $p<0.05$.

\section{Results}

The mean PASI score of patients enrolled in the study was 8.53 (range: 2.0-25.0). After the completion of the treatment, the mean PASI decreased to 4.74 (range: 0-18.2). The mean PASI improvement was 52\%. Table 2 presents the median, mean values and the range of plasma levels of the investigated parameter in the tested groups. The median of MMP-12 in the total group of psoriatic patients before the beginning of NBUVB phototherapy was significantly decreased when compared to healthy individuals. After the division of the total group based on disease severity, we found significantly lower concentrations of MMP-12 in patients with a mild and medium severity $(\mathrm{ng} / \mathrm{ml})$ compared to those in the control group. Additionally, after the division of the mild group based on PASI score, we detected significantly decreased levels of macrophage metalloelastase in subgroup la and subgroup Ib when compared to healthy individuals. There were no significant differences in MMP-12 concentrations between the total mild group, its subgroups and the total medium group.

Table 2. Plasma levels of the tested parameter in psoriatic patients and in the control group

\begin{tabular}{|c|c|c|c|c|}
\hline Group & Groups tested & Parameter & $\begin{array}{c}\text { MMP-12 [ng/ml] } \\
\text { before the treatment }\end{array}$ & $\begin{array}{l}\text { MMP-12 [ng/ml] } \\
\text { after the treatment }\end{array}$ \\
\hline & & & a & a \\
\hline \multirow{19}{*}{$\begin{array}{l}\text { Psoriatic } \\
\text { patients }\end{array}$} & Total group & Median & 3.19 & 2.63 \\
\hline & & Mean & 3.99 & 3.77 \\
\hline & & Range & $1.05-12.08$ & $0.10-13.64$ \\
\hline & & & $a, b$ & a \\
\hline & Mild & Median & 3.51 & 2.60 \\
\hline & & Mean & 4.18 & 3.89 \\
\hline & & Range & $1.05-12.08$ & $0.10-13.64$ \\
\hline & & & a & a \\
\hline & Mild - Subgroup la & Median & 3.58 & 3.27 \\
\hline & & Mean & 3.95 & 3.78 \\
\hline & & Range & $1.42-12.08$ & 1.09-13.64 \\
\hline & & & $a, b$ & a \\
\hline & Mild - Subgroup Ib & Median & 3.44 & 2.36 \\
\hline & & Mean & 4.37 & 3.99 \\
\hline & & Range & $1.05-11.01$ & $0.10-11.88$ \\
\hline & Medium & & a & a \\
\hline & & Median & 2.395 & 2.63 \\
\hline & & Mean & 3.57 & 3.49 \\
\hline & & Range & $1.196-10.68$ & $0.95-11.36$ \\
\hline \multirow{3}{*}{$\begin{array}{l}\text { Control } \\
\text { group }\end{array}$} & Healthy subjects & Median & \multirow{3}{*}{\multicolumn{2}{|c|}{$\begin{array}{c}6.02 \\
6.796 \\
1.01-13.54\end{array}$}} \\
\hline & & Mean & & \\
\hline & & Range & & \\
\hline
\end{tabular}

aStatistically significant when psoriatic patients are compared with healthy subjects; ${ }^{b}$ statistically significant when pretreatment concentration are compared to post-treatment levels. 
The Spearman rank correlation was used in the dependence analyses of the investigated parameter. We observed no associations between the concentrations of the analyzed parameters and disease severity, age, gender or duration of psoriatic disease.

The course of NBUVB phototherapy changed the concentrations of the tested enzyme. After 20 radiations, the plasma levels of MMP-12 were lower than before the commencement of the treatment but this change was not statistically significant when compared to pretreatment values. Furthermore, we noticed significantly declined concentrations of the chosen enzyme in patients with mild disease advancement, but not in the medium group. Moreover, based on PASI score we found significantly decreased plasma levels of MMP-12 in subgroup Ib. The post-treatment value of MMP-12 was significantly lower in the total group and all subgroups when compared to control cases. There were no statistically significant differences in macrophage metalloelastase concentrations between the total mild group, its subgroups and the medium group after the NBUVB therapy course.

\section{Discussion}

Matrix metalloproteinase 12 was detected and described as an enzyme secreted by inflammatory macrophages [12]. MMP-12 presents a various substrate specificity and is able to degrade matrix components such as: recognizing elastin, type IV collagen, laminins, entactin, vitronectin, $\alpha_{1}$-antitrypsin and fibronectin. Moreover, many studies demonstrated the implication of macrophage metalloelastase in a large number of inflammatory and degenerative conditions, including atherosclerosis, chronic pulmonary inflammation and fibrosis, cancers, skin and kidney diseases. Increased concentrations of MMP-12 were detected also in the serum of patients suffering from systemic sclerosis [13-15].

An important aim of this study was to evaluate the plasma levels of MMP-12 in psoriatic patients depending on disease advancement.

During psoriasis eruption, cellular composition of the epidermis is significantly modified by infiltrating immune cells. In fact, nearly 20 million of the total 28-30 million body lymphocytes migrate to the sites of damage. The following are present in the dermis: an inflammatory infiltrate composed of lymphocytes, macrophages, mast cells and neutrophils [4, 16]. Disturbed synthesis of MMPs may result from the abnormal cytokine production by infiltrating inflammatory cells (e.g. TNF- $\alpha$, IL-1, IFN- $\gamma$, IL-6, TGF- $\alpha$, EGF) [17].

As regards available literature, we found no papers on the concentrations of MMP-12 in the serum or plasma of psoriatic patients. Suomela et al. demonstrated that MMP-12 was expressed in psoriatic lesions, but not in non-lesional skin [18]. Their observations are in line with those performed by Starodubtseva et al. [19]. There have been a few studies describing other metalloproteinases in psoriatic patients. For instance, Flisiak et al. detected significantly increased levels of MMP-1 in the plasma of psoriatic patients compared to healthy controls [20]. Significantly higher serum concentrations of MMP-3 and MMP-9 in psoriatic patients were also observed by other authors [21, 22].

The onset and development of psoriasis is commenced with the processes of angiogenesis in the superficial dermal microvasculature. Dermal papillary capillaries are prominently elongated and show increased tortuosity, dilatation and permeability. These morphological disorders appear prior to those visible on the epidermis [14]. Interestingly, it has been shown that MMP-12 is capable of transforming plasminogen into angiostatin, a crucial inhibitor of endothelial cell proliferation and angiogenesis. In fact, the overexpression of MMP-12 seems to correlate with decreased angiogenesis and vascular invasion in cancer, and suppress tumor angiogenesis in animal models [23, 24].

In the view of this commonly known information, our findings seem to be vital. According to our study, macrophage metalloelastase concentrations were significantly decreased compared to healthy individuals, and they declined with the increase in disease severity. These data indicate that the more intensive angiogenesis, the more extended the psoriatic scales [15].

The alteration of the cytokine profile, induction of apoptosis and immunosuppression promotion are the most known mechanisms determining the usefulness and effectiveness of NBUVB phototherapy in the treatment of psoriasis and many other skin disorders [10, 25]. Furthermore, clinical improvement of psoriasis by NBUVB is connected with the downregulation of IL-17 pathway, also type I and type II interferon (IFN) signaling pathways - the processes which are critical in the pathogenesis of the disease [26]. The data obtained by Racz et al. showed that clinically effective NBUVB therapy was also based on the suppression of a wide range of important molecular pathways in psoriatic skin $[27,28]$.

Our research demonstrated that the NBUVB phototherapy course decreased the concentration of MMP-12, but this change was not statistically significant in the total group. Probably, the absence of that significance was caused by the lack of patients with advanced psoriasis in the analyzed group. The reason determining this kind of cohort selection is that NBUVB phototherapy is recommended, used and found effective, especially in case of people with a mild and moderate disease. The division of the total group based on disease advancement revealed that macrophage metalloelastase levels decreased statistically significantly in patients with a mild disease advancement when comparing post-treatment to pre-treatment values. This information suggested that 
patients with mild lesions are most sensitive to this kind of treatment.

Our observations indicate that MMP-12 seems to be a negatively reacting plasma biomarker of psoriasis. If so, after NBUVB therapy, because the disease is ameliorated, MMP-12 levels should be up-regulated. However opposite results were shown in the study. The reason explaining this fact is time of blood collection. We collected samples shortly after last NBUVB treatment, which is connected with a decrease in plasma MMP-12 due to the acute cell death induced by NBUVB. There is a big possibility that during the chronic phase, MMP-12 levels would bounce back.

As we mentioned before, there are no previous works on MMP-12 levels in psoriatic patients and the influence of any kind of treatment on that value. Flisiak et al. analyzed the influence of a typical application of $5 \%$ salicyl treatment, and stated that such therapy resulted in a decrease in MMP-1 levels of psoriatic patients [29]. The same tendency has been demonstrated by another research team who observed that UVA phototherapy caused the decrease in MMP-1 concentration [30].

\section{Conclusions}

Decreased synthesis of MMP-12 may lead to the stimulation of the epidermal angiogenesis process, which results in the appearance and spread of psoriatic scales. According to this information, MMP-12 seems to be a negatively reacting plasma biomarker of the studied disease. In view of insufficient bibliographic data available, this study needs to be expanded to scale concentrations of the analyzed enzyme, also blood examinations in the chronic phase after NBUVB treatment and thus it is our further research plan to prove the theory presented in this paper.

\section{Acknowledgments}

This work was supported by a grant from the Medical University of Białystok (154-30589F).

\section{Conflict of interest}

The authors declare no conflict of interest.

\section{References}

1. Alexis AF, Blackcloud P. Psoriasis in skin of color: epidemiology, genetics, clinical presentation, and treatment nuances. J Clin Aesthet Dermatol 2014; 7: 16-24.

2. Queiro R, Tejón P, Alonso S, et al. Age at disease onset: a key factor for understanding psoriatic disease. Rheumatology (Oxford) 2014; 53: 1178-85.

3. Kim GE, Seidler E, Kimball AB. A measure of chronic quality of life predicts socioeconomic and medical outcomes in psoriasis patients. J Eur Acad Dermatol Venereol 2015; 29: 249-54
4. Ayala-Fontánez N, Soler DC, McCormick TS. Current knowledge on psoriasis and autoimmune diseases. Psoriasis Targets Ther 2016; 6: 7-32.

5. Huerta C, Rivero E, Rodríguez LA. Incidence and risk factors for psoriasis in the general population. Arch Dermatol 2007; 143: 1559-65.

6. Mezentsev A, Nikolaev A, Bruskin S. Matrix metalloproteinases and their role in psoriasis. Gene 2014; 540: 1-10.

7. Ravi Kanth VV, Nageshwar Reddy D. Role of matrix metalloproteinases in physiological processes and disease. Indian J Med Res 2014; 140: 585-7.

8. Ławicki S, Głażewska EK, Sobolewska M, et al. Plasma levels and diagnostic utility of macrophage colony-stimulating factor, matrix metalloproteinase-9, and tissue inhibitor of metalloproteinases-1 as new biomarkers of breast cancer. Ann Lab Med 2016; 36: 223-9.

9. Zajkowska M, Ławicki S, Głażewska E, et al. Plasma levels and diagnostic utility of VEGF, MMP-9, and TIMP-1 in the diagnosis of patients with breast cancer. OncoTargets Therapy 2016; 9: 911-9.

10. Racz E, Prens EP. Phototherapy and photochemotherapy for psoriasis. Dermatol Clin 2015; 33: 79-89.

11. Fredriksson T, Pettersson U. Severe psoriasis oral therapy with a new retinoid. Dermatologica 1978; 157: 238-44.

12. Banda MJ, Werb Z. Mouse macrophage elastase. Purification and characterization as a metalloproteinase. Biochem J 1981; 193: 589-605.

13. Sternlicht MD, Werb Z. How matrix metalloproteinases regulate cell behavior. Annu Rev Cell Dev Biol 2001; 17: 463-516.

14. Gronski TJ, Martin RL, Kobayashi DK, et al. Hydrolysis of a broad spectrum of extracellular matrix proteins by human macrophage elastase. J Biol Chem 1997; 272: 12189-94.

15. Manetti M, Guiducci S, Romano E. Increased serum levels and tissue expression of matrix metalloproteinase- 12 in patients with systemic sclerosis: correlation with severity of skin and pulmonary fibrosis and vascular damage. Ann Rheum Dis 2012; 71: 1064-72.

16. Heidenreich R, Röcken M, Ghoreschi K. Angiogenesis drives psoriasis pathogenesis. Int J Exp Pathol 2009; 90: 232-48.

17. Eysteinsdóttir JH, Sigurgeirsson B, Ólafsson JH, et al. The role of Th17/Tc17 peripheral blood T cells in psoriasis and their positive therapeutic response. Scand I Immunol 2013; 78: 529-37.

18. Suomela S, Kariniemi AL, Snellman E, Saarialho-Kere U. Metalloelastase (MMP-12) and 92-kDa gelatinase (MMP-9) as well as their inhibitors, TIMP-1 and -3, are expressed in psoriatic lesions. Exp Dermatol 2001; 10: 175-83.

19. Starodubtseva NL, Sobolev VV, Soboleva AG, et al. Expression of genes for metalloproteinases (MMP-1, MMP-2, MMP-9, and MMP-12) associated with psoriasis. Genetika 2011; 47: 1254-61.

20. Flisiak I, Porebski P, Chodynicka B. Effect of psoriasis activity on metalloproteinase-1 and tissue inhibitor of metalloproteinase-1 in plasma and lesional scales. Acta Derm Venereol 2006; 86: 17-21.

21. Altrichter S, Boodstein N, Maurer M. Matrix metalloproteinase-9: a novel biomarker for monitoring disease activity in patients with chronic urticaria patients? Allergy 2009; 64: 652-6.

22. Chandran Y, Cook RJ, Edwin J, et al. Soluble biomarkers differentiate patients with psoriatic arthritis from those with psoriasis without arthritis. Rheumatology 2010; 49: 1399405. 
23. Vaalamo M, Kariniemi AL, Shapiro SD, et al. Enhanced expression of human metalloelastase (MMP-12) in cutaneous granulomas and macrophage migration. J Invest Dermatol 1999; 112: 499-505.

24. Yang W, Arii S, Gorrin-Rivas MJ, et al. Human macrophage metalloelastase gene expression in colorectal carcinoma and its clinicopathologic significance. Cancer 2001; 91: 1277-83.

25. Reich A, Mędrek K. Effects of narrow band UVB (311 nm) irradiation on epidermal cells. Int J Mol Sci 2013; 14: 8456-66.

26. Wasilewska A, Winiarska M, Olszewska M, Rudnicka L. Interleukin-17 inhibitors. A new era in treatment of psoriasis and other skin diseases. Adv Dermatol Allergol 2016; 33: 247-52.

27. Rácz E, Prens EP, Kurek D, et al. Effective treatment of psoriasis with narrow-band UVB phototherapy is linked to suppression of the IFN and Th17 pathways. J Invest Dermatol 2011; 131: 1547-58.

28. Richard EG, Hönigsmann H. Phototherapy, psoriasis, and the age of biologics. Photodermatol Photoimmunol Photomed 2014; 30: 3-7.

29. Flisiak I, Myśliwiec H, Chodynicka B. Effect of psoriasis treatment on plasma concentrations of metalloproteinase- 1 and tissue inhibitor of metalloproteinases-1. J Eur Acad Dermatol Venereol 2005; 19: 418-21.

30. Wolk K, Witte E, Wallace E, et al. IL-22 regulates the expression of genes responsible for antimicrobial defense, cellular differentiation, and mobility in keratinocytes: a potential role in psoriasis. Eur J Immunol 2006; 36: 1309-23. 